\title{
Analysis of Stochastic Gilpin-Ayala Competition System
}

\author{
Lei Liu' ${ }^{1}$ and Quanxin $\mathrm{Zhu}^{2}$ \\ ${ }^{1}$ College of Science, Hohai University, Nanjing 210098, China \\ ${ }^{2}$ School of Mathematical Sciences and Institute of Finance and Statistics, Nanjing Normal University, Nanjing 210023, China
}

Correspondence should be addressed to Lei Liu; liulei_hust@163.com

Received 4 August 2014; Accepted 4 September 2014; Published 5 November 2014

Academic Editor: Ramachandran Raja

Copyright (C) 2014 L. Liu and Q. Zhu. This is an open access article distributed under the Creative Commons Attribution License, which permits unrestricted use, distribution, and reproduction in any medium, provided the original work is properly cited.

\begin{abstract}
This paper is concerned with the asymptotic behavior for stochastic Gilpin-Ayala competition system. The sufficient conditions for existence of stationary distribution and extinction are established. And a certain asymptotic property of the solution is also obtained. A nontrivial example is provided to illustrate our results.
\end{abstract}

\section{Introduction}

One of the most common phenomena considering ecological population is that many species which grow in the same environment compete for the limited resources or in some way inhibit others' growth. It is therefore very important to study the competition models for multispecies. It is well known that one of the famous models is the following classical Lotka-Volterra competition system:

$$
\frac{d x_{i}}{d t}=x_{i}\left(b_{i}-\sum_{j=1}^{n} a_{i j} x_{j}\right), \quad i=1, \ldots, n,
$$

where $x_{i}(t)$ represents the population size of species $i$ at time $t$, the constant $b_{i}$ is the growth rate of species $i$, and $a_{i j}$ represents the effect of interspecific $(i \neq j)$ or intraspecific $(i=j)$ interaction. The Lotka-Volterra models have often been severely criticized. One disadvantage of Lotka-Volterra models is that in such a model, the rate of change in the density of each species is a linear function of densities of the interacting species. In order to yield significantly more accurate results, Gilpin and Ayala proposed the the following Gilpin-Ayala models; detailed studies related to the model may be found in [1]:

$$
\frac{d x_{i}}{d t}=x_{i}\left(b_{i}-a_{i i} x_{i}^{\theta_{i}}-\sum_{j \neq i} a_{i j} x_{j}\right), \quad i=1, \ldots, n,
$$

where $\theta_{i}$ are the parameters to modify the classical LotkaVolterra model.
On the other hand, population systems are inevitably affected by environmental noise. It is therefore useful to reveal how the noise affects the population systems. Recall that the parameter $b_{i}$ in (2) represents the intrinsic growth rate of the population. In practice we usually estimate it by an average value plus an error which follows a normal distribution; then the intrinsic growth rate becomes

$$
b_{i} \longrightarrow b_{i}+\sigma_{i} \dot{B}_{i}(t)
$$

where $B_{i}(t)(i=1, \ldots, n)$ are Brown motions with $B_{i}(0)=$ 0 and $\sigma_{i}^{2}$ represent the intensities of the noise. As a result, system (2) becomes the stochastic Gilpin-Ayala system as follows:

$$
\begin{array}{r}
d x_{i}=x_{i}\left(b_{i}-a_{i i} x_{i}^{\theta_{i}}-\sum_{j \neq i} a_{i j} x_{j}\right) d t+\sigma_{i} x_{i} d B_{i}(t), \\
i=1, \ldots, n,
\end{array}
$$

and we impose the following condition:

$$
\begin{array}{r}
\theta_{i}>1, \quad a_{i i}>0, \quad a_{i j} \geq 0, \\
1 \leq i, \quad j \leq n, \quad i \neq j .
\end{array}
$$

The stochastic Lotka-Volterra model has been extensively studied due to its universal existence and importance; see [210]. More recently, the existence of stationary distribution and extinction of stochastic Lotka-Volterra system have received 
a lot of attention, which can give a good explanation of the recurring phenomena in population system. Under what conditions can a stochastic Lotka-Volterra system has a stationary distribution? It is an open topic until very recently Mao [11] gave a positive answer. Since then, this topic has received a lot of attention; the readers are referred to [1114]. In addition, the asymptotic behavior of $\log x_{i}(t) / t, i=$ $1, \ldots, n$ for various stochastic Lotka-Volterra systems has been considered by many authors $[4,5,10,12]$, which is an important and useful property on asymptotic estimation for corresponding population systems.

However, these properties for stochastic Gilpin-Ayala system (4) have not been investigated, which remain an interesting research topic. We aim to establish new results on these properties for system (4). It is well known that the stochastic Gilpin-Ayala system (4) is a highly nonlinear system; the method for classic Lotka-Volterra system cannot be directly applied to system (4). By the Lyapunov methods, and some techniques to deal with the nonquadratic item, sufficient criteria are established which ensure the existence of a stationary distribution and extinction. By using some stochastic analysis techniques, an asymptotic property for system (4) is obtained.

\section{Notation}

Throughout this paper, unless otherwise specified, let $\left(\Omega, \mathscr{F},\left\{\mathscr{F}_{t}\right\}_{t \geq 0}, \mathbb{P}\right)$ be a complete probability space with a filtration $\left\{\mathscr{F}_{t}\right\}_{t \geq 0}$ satisfying the usual conditions (i.e., it is increasing and right continuous while $\mathscr{F}_{0}$ contains all $\mathbb{P}$-null sets). Let $B(t)=\left(B_{t}^{1}, \ldots, B_{t}^{n}\right)$ be a $n$-dimensional Brownian motion defined on the probability space. If $a$ and $b$ are real numbers, then $a \vee b$ denotes the maximum of $a$ and $b$, and $a \wedge b$ stands for the minimum of $a$ and $b$. If $A \in R^{n \times n}$ is symmetric, its largest and smallest eigenvalues are denoted by $\lambda_{\max }(A)$ and $\lambda_{\min }(A)$. Let $x^{*}=\left(x_{1}^{*}, \ldots, x_{n}^{*}\right)$ be the positive equilibrium of the deterministic Gilpin-Ayala competition system (2), that is, the solution of the following equation:

$$
b_{i}=a_{i i}\left(x_{i}^{*}\right)^{\theta_{i}}+\sum_{j \neq i} a_{i j} x_{j}^{*}, \quad i=1, \ldots, n .
$$

In the same way as Mao et al. [8] did, we can also show the following result on the existence of global positive solution.

Lemma 1. Assume that condition (5) holds. Then, for any given initial value $x_{0} \in R_{+}^{n}$, there is a unique solution $x\left(t, x_{0}\right)$ to system (4) and the solution will remain in $R_{+}^{n}$ with probability 1; namely,

$$
\mathbb{P}\left\{x\left(t, x_{0}\right) \in R_{+}^{n}, \forall t \geq 0\right\}=1,
$$

for any $x_{0} \in R_{+}^{n}$.

Lemma 2. Let condition (5) hold. Then, for any $p>0$ and any given initial value $x_{0} \in R_{+}^{n}$, there exists a constant $K_{p}$ such that

$$
\sup _{0 \leq t \leq \infty} E \sum_{i=1}^{n} x_{i}^{p}\left(t, x_{0}\right)<K_{p} .
$$

The proof of the lemma is rather standard so it is omitted.

\section{An Asymptotic Property}

The main aim of this section is to consider the large time behavior of $\log x_{i}(t) / t, i=1, \ldots, n$. To this end, we consider two auxiliary stochastic differential equations as follows:

$$
\begin{gathered}
d \varphi_{i}=\varphi_{i}\left(b_{i}-a_{i i} \varphi_{i}^{\theta_{i}}\right) d t+\sigma_{i} \varphi_{i} d B_{i}(t), \\
\varphi_{i}(0)=x_{i}(0), \quad i=1, \ldots, n, \\
d y_{i}=y_{i}\left(b_{i}-a_{i i} y_{i}^{\theta_{i}}-\sum_{j \neq i} a_{i j} \varphi_{j}\right) d t+\sigma_{i} y_{i} d B_{i}(t), \\
y_{i}(0)=x_{i}(0), \quad i=1, \ldots, n .
\end{gathered}
$$

Then it follows from comparison principle (see [15]) that

$$
y_{i}(t) \leq x_{i}(t) \leq \varphi_{i}(t), \quad i=1, \ldots, n .
$$

Lemma 3. Let condition (5) hold. Then the solution to system (9) has the following property:

$$
\frac{\log \varphi_{i}(t)}{t}=0, \quad i=1, \ldots, n \text {, a.s. }
$$

The proof is similar to Li et al. [5] and is omitted here.

Theorem 4. Let condition (5) hold and $x\left(t, x_{0}\right)$ be the global solution to system (4) with any positive initial value $x_{0}$. Assume moreover that

$$
\begin{array}{r}
b_{i}-\frac{\sigma_{i}^{2}}{2}>0, \quad b_{i}-\frac{\sigma_{i}^{2}}{2}-\sum_{j \neq i} a_{i j}\left(\frac{b_{j}-\left(\sigma_{j}^{2} / 2\right)}{a_{j j}}\right)_{j}^{1 / \theta_{j}}>0, \\
i=1, \ldots, n .
\end{array}
$$

Then the solution $x\left(t, x_{0}\right)$ of system (4) has the following property:

$$
\lim _{t \rightarrow \infty} \frac{\log x_{i}\left(t, x_{0}\right)}{t}=0, \quad i=1, \ldots, n, \text { a.s. }
$$

Proof. Let $x(t)$ be $x\left(t, x_{0}\right)$ for simplicity. By virtue of Lemma 3 and (11), we have $\lim \sup _{t \rightarrow \infty}\left(\log x_{i}(t) / t\right) \leq$ $0, i=1, \ldots, n$, a.s. Thus it remains to show that $\liminf _{t \rightarrow \infty}\left(\log x_{i}(t) / t\right) \geq 0, i=1, \ldots, n$, a.s. It is sufficient to show

$$
\liminf _{t \rightarrow \infty} \frac{\log y_{i}(t)}{t} \geq 0, \quad i=1, \ldots, n, \text { a.s. }
$$


By Ito's formula, $y_{i}(t)$ satisfies

$$
\begin{aligned}
& \frac{1}{y_{i}^{\theta_{i}}(t)}=\frac{1}{x_{i}^{\theta_{i}}(0)} \exp \left\{\left(\frac{\sigma_{i}^{2}}{2}-b_{i}\right) \theta_{i} t-\theta_{i} \sigma_{i} B_{i}(t)\right. \\
& \left.+\theta_{i} \sum_{j \neq i} \int_{0}^{t} a_{i j} \varphi_{j}(s) d s\right\} \\
& +a_{i i} \theta_{i} \int_{0}^{t} \exp \left\{\left(\frac{\sigma_{i}^{2}}{2}-b_{i}\right) \theta_{i}(t-s)\right. \\
& -\theta_{i} \sigma_{i}\left(B_{i}(t)-B_{i}(s)\right) \\
& \left.+\theta_{i} \sum_{j \neq i} \int_{s}^{t} a_{i j} \varphi_{j}(\tau) d \tau\right\} d s \\
& =: J_{i 1}+J_{i 2}, \quad i=1, \ldots, n .
\end{aligned}
$$

$$
\begin{aligned}
& :=k_{i}(t-s) \\
& +m_{i}\left(B_{i}(t)-B_{i}(s)\right) \\
& +d_{i}\left(\log \varphi_{i}(s)-\log \varphi_{i}(t)\right), \\
& \quad i=1, \ldots, n .
\end{aligned}
$$

The well-known Hölder inequality yields

$$
\begin{array}{r}
\int_{s}^{t} \varphi_{i}(\tau) d \tau \leq(t-s)^{1-1 / \theta_{i}} \\
\times\left(k_{i}(t-s)+m_{i}\left(B_{i}(t)-B_{i}(s)\right)\right. \\
\left.+d_{i}\left(\log \varphi_{i}(s)-\log \varphi_{i}(t)\right)\right)^{1 / \theta_{i}} \\
i=1, \ldots, n
\end{array}
$$

For $i=1, \ldots, n$, it follows from the inequality $(a+b+c)^{p} \leq$ $3^{(p-1) \vee 0}\left(a^{p}+b^{p}+c^{p}\right)$ that

$$
\begin{aligned}
\int_{s}^{t} \varphi_{i}(\tau) d \tau & \\
\leq(t-s)^{1-1 / \theta_{i}}\left(k_{i}^{\theta_{i}}(t-s)^{\theta_{i}}\right. & \\
& +m_{i}^{\theta_{i}}\left(B_{i}(t)-\min _{0 \leq s \leq t} B_{i}(s)\right)^{\theta_{i}} \\
& \left.+d_{i}^{\theta_{i}}\left(\max _{0 \leq s \leq t} \log \varphi_{i}(s)-\log \varphi_{i}(t)\right)^{\theta_{i}}\right)^{1 / \theta_{i}} .
\end{aligned}
$$

For $i=1, \ldots, n$, set

$$
\begin{aligned}
B_{i}^{*}(t) & :=B_{i}(t)-\min _{0 \leq s \leq t} B_{i}(s), \\
\xi_{i}(t) & :=\max _{0 \leq s \leq t} \log \varphi_{i}(s)-\log \varphi_{i}(t) .
\end{aligned}
$$

Substituting these inequalities into (16) yields

$$
\begin{aligned}
& J_{i 1} \leq \frac{1}{x_{i}^{\theta_{i}}(0)} \exp \left\{\left(\frac{\sigma_{i}^{2}}{2}-b_{i}\right) \theta_{i} t-\theta_{i} \sigma_{i} B_{i}(t)\right. \\
& +\theta_{i} \sum_{j \neq i} a_{i j} t^{1-1 / \theta_{j}} \\
& \times\left(k_{i} t+m_{i} B_{i}(t)\right. \\
& \left.\left.+d_{i}\left(\log \varphi_{i}(0)-\log \varphi_{i}(t)\right)\right)^{1 / \theta_{j}}\right\} \\
& \leq \frac{1}{x_{i}^{\theta_{i}}(0)} \exp \left\{\left(\frac{\sigma_{i}^{2}}{2}-b_{i}\right) \theta_{i} t\right. \\
& +\theta_{i} \sigma_{i}\left(\max _{0 \leq s \leq t} B_{i}(s)-B_{i}(t)\right) \\
& +\theta_{i} \sum_{j \neq i} a_{i j} t^{1-1 / \theta_{j}} \\
& \times\left(\left(k_{j}^{1 / \theta_{j}}(t-s)^{1 / \theta_{j}}\right.\right. \\
& +m_{j}^{1 / \theta_{j}}\left(B_{j}^{*}(t)\right)^{1 / \theta_{j}} \\
& \left.\left.\left.+d_{j}^{1 / \theta_{j}}\left(\xi_{j}(t)\right)^{1 / \theta_{j}}\right)\right)\right\}
\end{aligned}
$$




$$
\begin{aligned}
& \leq \frac{1}{x_{i}^{\theta_{i}}(0)} \exp \{ \theta_{i}\left(\frac{\sigma_{i}^{2}}{2}-b_{i}+\sum_{j \neq i} a_{i j} k_{j}^{1 / \theta_{j}}\right) t \\
&+\theta_{i} \sigma_{i}\left(\max _{0 \leq s \leq t} B_{i}(s)-B_{i}(t)\right) \\
&+\theta_{i} \sum_{j \neq i} t^{1-1 / \theta_{j}} a_{i j} \\
& \times\left(\left(m_{j}^{1 / \theta_{j}}\left(B_{j}^{*}(t)\right)^{1 / \theta_{j}}\right.\right. \\
&\left.\left.\left.+d_{j}^{1 / \theta_{j}}\left(\xi_{j}(t)\right)^{1 / \theta_{j}}\right)\right)\right\} d s \\
& i=1, \ldots, n .
\end{aligned}
$$$$
\leq a_{i i} \theta_{i} \int_{0}^{t} \exp \left\{\theta_{i}\left(\frac{\sigma_{i}^{2}}{2}-b_{i}+\sum_{j \neq i} a_{i j} k_{j}^{1 / \theta_{j}}\right)\right.
$$$$
\times(t-s)+\theta_{i} \sigma_{i}\left(\max _{0 \leq s \leq t} B_{i}(s)-B_{i}(t)\right)
$$$$
+\theta_{i} \sum_{j \neq i} t^{1-1 / \theta_{j}} a_{i j}
$$$$
\times\left(\left(m_{j}^{1 / \theta_{j}}\left(B_{j}^{*}(t)\right)^{1 / \theta_{j}}\right.\right.
$$$$
\left.\left.\left.+d_{j}^{1 / \theta_{j}}\left(\xi_{j}(t)\right)^{1 / \theta_{j}}\right)\right)\right\} d s
$$

Similarly, we get

$$
\left.\begin{array}{rl}
J_{i 2} \leq a_{i i} \theta_{i} \int_{0}^{t} \exp \left\{\left(\frac{\sigma_{i}^{2}}{2}-b_{i}\right) \theta_{i}(t-s)\right. & \\
& -\theta_{i} \sigma_{i}\left(B_{i}(t)-B_{i}(s)\right) \\
& +\theta_{i} \sum_{j \neq i}(t-s)^{1-1 / \theta_{j}} \\
\times\left(k_{j}(t-s)\right. & +m_{j}\left(B_{j}(t)-B_{j}(s)\right) \\
+d_{j}\left(\log \varphi_{j}(s)\right. & \left.\left.-\log \varphi_{j}(t)\right)\right)^{1 / \theta_{j}}
\end{array}\right\} d s
$$$$
\leq a_{i i} \theta_{i} \int_{0}^{t} \exp \left\{\left(\frac{\sigma_{i}^{2}}{2}-b_{i}\right) \theta_{i}(t-s)\right.
$$$$
\begin{gathered}
+\theta_{i} \sigma_{i}\left(\max _{0 \leq s \leq t} B_{i}(s)-B_{i}(t)\right) \\
+\theta_{i} \sum_{j \neq i}(t-s)^{1-1 / \theta_{j}} a_{i j} \\
\times\left(\left(k_{j}^{1 / \theta_{j}}(t-s)^{1 / \theta_{j}}\right.\right. \\
+m_{j}^{1 / \theta_{j}}\left(B_{j}^{*}(t)\right)^{1 / \theta_{j}}
\end{gathered}
$$$$
\left.\left.\left.+d_{j}^{1 / \theta_{j}}\left(\xi_{j}(t)\right)^{1 / \theta_{j}}\right)\right)\right\} d s
$$

Substituting (21) and (22) into (16) yields

$$
\begin{aligned}
& \frac{1}{y_{i}^{\theta_{i}}(t)} \leq\left(\frac{1}{x_{i}^{\theta_{i}}(0)} \exp \left\{\theta_{i}\left(\frac{\sigma_{i}^{2}}{2}-b_{i}+\sum_{j \neq i} a_{i j} k_{j}^{1 / \theta_{j}}\right) t\right\}\right. \\
& +\theta_{i} a_{i i} \int_{0}^{t} \exp \left\{\theta_{i}\left(\frac{\sigma_{i}^{2}}{2}-b_{i}+\sum_{j \neq i} a_{i j} k_{j}^{1 / \theta_{j}}\right)\right. \\
& \times(t-s)\} d s) \\
& \times \exp \left\{\theta_{i} \sigma_{i}\left(\max _{0 \leq s \leq t} B_{i}(s)-B_{i}(t)\right)\right. \\
& +\theta_{i} \sum_{j \neq i} t^{1-1 / \theta_{j}}\left(\left(m_{j}^{1 / \theta_{j}}\left(B_{j}^{*}(t)\right)^{1 / \theta_{j}}\right.\right. \\
& \left.\left.\left.+d_{j}^{1 / \theta_{j}}\left(\xi_{j}(t)\right)^{1 / \theta_{j}}\right)\right)\right\} \\
& :=Z_{i}^{-1}(t) \exp \left\{\theta_{i} \sigma_{i}\left(\max _{0 \leq s \leq t} B_{i}(s)-B_{i}(t)\right)\right. \\
& +\theta_{i} \sum_{j \neq i} t^{1-1 / \theta_{j}}\left(\left(m_{j}^{1 / \theta_{j}}\left(B_{j}^{*}(t)\right)^{1 / \theta_{j}}\right.\right. \\
& \left.\left.\left.+d_{j}^{1 / \theta_{j}}\left(\xi_{j}(t)\right)^{1 / \theta_{j}}\right)\right)\right\}, \\
& i=1, \ldots, n \text {, }
\end{aligned}
$$


where $Z_{i}(t)$ is the solution of the following system:

$$
\begin{gathered}
\dot{Z}_{i}(t)=\theta_{i} Z_{i}\left(b_{i}-\frac{\sigma_{i}^{2}}{2}-\sum_{j \neq i} a_{i j} k_{j}^{1 / \theta_{j}}-a_{i i} Z_{i}(t)\right), \\
Z_{i}(0)=x_{i}(0), \quad i=1, \ldots, n .
\end{gathered}
$$

A simple computation shows that

$$
\begin{aligned}
& \frac{\log y_{i}^{\theta_{i}}(t)}{t} \geq-\frac{\log Z_{i}(t)}{t} \\
&-\theta_{i} \sigma_{i} \frac{\left(\max _{0 \leq s \leq t} B_{i}(s)-B_{i}(t)\right)}{t} \\
&-\sum_{n \neq j} a_{i j}\left(\frac{m_{j} B_{j}^{*}(t)}{t}\right)^{1 / \theta_{j}} \\
&-\sum_{n \neq j} a_{i j}\left(\frac{d_{j}\left(\xi_{j}(t)\right)}{t}\right)^{1 / \theta_{j}}, \\
& i=1, \ldots, n .
\end{aligned}
$$

Using the property of Brownian motion, we conclude that

$$
\begin{gathered}
\lim _{t \rightarrow \infty} \frac{\left(\max _{0 \leq s \leq t} B_{i}(s)-B_{i}(t)\right)}{t}=0, \\
\lim _{t \rightarrow \infty} \frac{\left(B_{j}(t)-\min _{0 \leq s \leq t} B_{j}(s)\right)}{t}=0, \\
i=1, \ldots, n, \text { a.s. }
\end{gathered}
$$

It is easy to see that if $b_{i}-\sigma_{i}^{2} / 2-\sum_{j \neq i} a_{i j} k_{j}^{1 / \theta_{j}}>0$, then we have

$$
\lim _{t \rightarrow \infty} \frac{\log Z_{i}(t)}{t}=0, \quad i=1, \ldots, n, \text { a.s. }
$$

Besides, it follows from Lemma 3 that

$$
\begin{array}{r}
\lim _{t \rightarrow \infty} \frac{\left(\max _{0 \leq s \leq t} \log \varphi_{j}(s)-\log \varphi_{j}(t)\right)}{t}=0, \\
i=1, \ldots, n, \quad \text { a.s. }
\end{array}
$$

The required assertion (15) follows by letting $t \rightarrow \infty$ on both sides of (25) and using conditions (26)-(28). The proof is therefore completed.

\section{Stationary Distribution}

The main aim of this section is to study the existence of a unique stationary distribution of the system (4). Let us prepare a known lemma (see Hasminskii [16, pp. 106-125]). Let $X(t)$ be a homogeneous Markov process in $E^{n} \subset R^{n}$ described by the following stochastic differential equation:

$$
d X(t)=b(X) d t+\sum_{m=1}^{d} \sigma_{m}(X) d B_{m}(t) .
$$

The diffusion matrix is

$$
A(x)=\left(a_{i j}(x)\right), \quad a_{i j}(x)=\sum_{m=1}^{d} \sigma_{m}^{i}(x) \sigma_{m}^{j}(x) .
$$

To be more precise, let $P_{x_{0}, t}$ denote the probability measure induced by $X\left(t, x_{0}\right)$, that is

$$
P_{x_{0}, t}(A)=\mathbb{P}\left(X\left(t, x_{0}\right) \in A\right), \quad A \in \mathscr{B}\left(E^{n}\right),
$$

where $\mathscr{B}\left(E^{n}\right)$ is the $\sigma$-algebra of all the Borel sets $A \subset E^{n}$.

Lemma 5 (see [16]). We assume that there is a bounded open subset $G \subset E^{n}$ with a regular (i.e., smooth) boundary such that its closure $\bar{G} \subset E^{n}$, and consider the following:

(i) in the domain $G$ and some neighborhood, therefore, the smallest eigenvalue of the diffusion matrix $A(x)$ is bounded away from zero;

(ii) if $x \in E^{n} \backslash G$, the mean time $\tau$ at which a path issuing from $x$ reaches the set $G$ is finite, and $\sup _{x \in K} E_{x} \tau<+\infty$ for every compact subset $K \in E^{n}$. And throughout this paper one sets inf $\emptyset=\infty$.

We then have the following assertions.

(1) The Markov process $X(t)$ has a stationary distribution $\mu(\cdot)$ with density in $E^{n}$, such that, for any borel set $B C$ $E^{n}$,

$$
\lim _{t \rightarrow \infty} \int_{B} f(y) P_{x_{0}, t}(d y)=\int_{B} f(y) \mu(d y) .
$$

(2) (ergodic property) Let $f(x)$ be a function integrable with respect to the measure $\mu(\cdot)$. Then

$$
\begin{aligned}
\mathbb{P}\left\{\lim _{t \rightarrow \infty} \frac{1}{t} \int_{0}^{t} f(x(s)) d s\right. \\
\left.=\int_{E^{n}} f(y) \mu(d y)\right\}=1 .
\end{aligned}
$$

Remark 6. The proof is given by [16] in detail. Exactly, the existence of stationary distribution with density is referred to Theorem 4.3 on page 117 while ergodic property (33) is referred to Theorem 4.2, page 110 .

Theorem 7. Let condition (5) hold and $x\left(t, x_{0}\right)$ be the global solution to system (4) with any positive initial value $x_{0}$. Assume that there exists $c=\left(c_{1}, \ldots, c_{n}\right) \gg 0$ such that

$$
\left(c_{i}\left(x^{*}\right)^{\theta_{i}-1} a_{i i}-\frac{1}{2} \sum_{i \neq j}\left(c_{i} a_{i j}+c_{j} a_{j i}\right)\right)>0,
$$

$$
\begin{array}{r}
\frac{1}{2} \sum_{k=1}^{n} c_{i} x_{i}^{*} \sigma_{i}^{2} \\
<\min _{1 \leq i \leq n}\left\{\left(x_{i}^{*}\right)^{2}\left(c_{i}\left(x^{*}\right)^{\theta_{i}-1} a_{i i}-\sum_{i \neq j}\left(c_{i} a_{i j}+c_{j} a_{j i}\right)\right)\right\}, \\
i=1, \ldots, n .
\end{array}
$$


Then there is a stationary distribution for system (4) and it has the ergodic property.

Proof. By Lemma 5, it suffices to prove that there exists some neighborhood $U$ and a nonnegative $C^{2}$-function $V(x)$ such that the diffusion matrix $H(x)=\operatorname{diag}\left(\sigma_{1} x_{1}, \ldots, \sigma_{n} x_{n}\right)$ is uniformly elliptical in $U$ and, for any $x \in R_{+}^{n} \backslash U, \mathscr{L} V(x)$ is negative (for details refer to [11]).

Applying Itô's formula to $V(x)=\sum_{i=1}^{n} c_{i}\left(x_{i}-x_{i}^{*}-\right.$ $\left.x_{i}^{*} \log \left(x_{i} / x_{i}^{*}\right)\right)$ yields

$$
\begin{aligned}
\mathscr{L} V(x)=\sum_{i=1}^{n}\left(c_{i} a_{i i}\left(x_{i}-x_{i}^{*}\right)\left(x_{i}^{\theta_{i}}-\left(x_{i}^{*}\right)^{\theta_{i}}\right)\right. \\
\left.+\sum_{i \neq j}^{n} c_{i} a_{i j}\left(x_{j}^{*}-x_{j}\right)\left(x_{i}^{*}-x_{i}\right)\right) \\
+\frac{1}{2} \sum_{i=1}^{n} c_{i} \sigma_{i}^{2} x_{i}^{*} .
\end{aligned}
$$

If $x_{i}>x_{i}^{*}$, since $\left(x_{i} / x_{i}^{*}\right)^{\theta_{i}} \geq\left(x_{i} / x_{i}^{*}\right)$, for $\theta_{i} \geq 1$, then

$$
\begin{aligned}
& \left(x_{i}-x_{i}^{*}\right)\left(x_{i}^{\theta_{i}}-\left(x_{i}^{*}\right)^{\theta_{i}}\right) \\
& =\left(x_{i}^{*}\right)^{\theta_{i}+1}\left(\frac{x_{i}}{x_{i}^{*}}-1\right)\left(\left(\frac{x_{i}}{x_{i}^{*}}\right)^{\theta_{i}}-1\right) \\
& \geq\left(x_{i}^{*}\right)^{\theta_{i}+1}\left(\frac{x_{i}}{x_{i}^{*}}-1\right)\left(\left(\frac{x_{i}}{x_{i}^{*}}\right)-1\right) \\
& =\left(x_{i}^{*}\right)^{\theta_{i}-1}\left(x_{i}-x_{i}^{*}\right)^{2} .
\end{aligned}
$$

If $x_{i}<x_{i}^{*}$, then

$$
\begin{aligned}
& \left(x_{i}-x_{i}^{*}\right)\left(x_{i}^{\theta_{i}}-\left(x_{i}^{*}\right)^{\theta_{i}}\right) \\
& =\left(x_{i}^{*}\right)^{\theta_{i}+1}\left(1-\frac{x_{i}}{x_{i}^{*}}\right)\left(1-\left(\frac{x_{i}}{x_{i}^{*}}\right)^{\theta}\right) \\
& \geq\left(x_{i}^{*}\right)^{\theta_{i}+1}\left(1-\frac{x_{i}}{x_{i}^{*}}\right)\left(1-\left(\frac{x_{i}}{x_{i}^{*}}\right)\right) \\
& =\left(x_{i}^{*}\right)^{\theta_{i}-1}\left(x_{i}-x_{i}^{*}\right)^{2} .
\end{aligned}
$$

Substituting (37) and (38) into (36) yields

$$
\begin{aligned}
\mathscr{L} V(x) \leq & -\sum_{i=1}^{n}\left(x_{i}^{*}\right)^{\theta_{i}-1} c_{i} a_{i i}\left(x_{i}-x_{i}^{*}\right)^{2} \\
& +\sum_{i=1}^{n} \sum_{i \neq j}^{n} c_{i} a_{i j}\left(x_{j}^{*}-x_{j}\right)\left(x_{i}^{*}-x_{i}\right) \\
& +\frac{1}{2} \sum_{i=1}^{n} c_{i} \sigma_{i}^{2} x_{i}^{*} .
\end{aligned}
$$

By the inequality $a b \leq(1 / 2)\left(a^{2}+b^{2}\right)$, we have

$$
\begin{aligned}
\mathscr{L} V(x) \leq & -\sum_{i=1}^{n}\left(x_{i}^{*}\right)^{\theta_{i}-1} c_{i} a_{i i}\left(x_{i}-x_{i}^{*}\right)^{2} \\
& +\sum_{i=1}^{n} \sum_{i \neq j}^{n} c_{i} a_{i j}\left(x_{j}^{*}-x_{j}\right)\left(x_{i}^{*}-x_{i}\right)+\frac{1}{2} \sum_{i=1}^{n} c_{i} \sigma_{i}^{2} x_{i}^{*} \\
\leq & -\sum_{i=1}^{n}\left(x_{i}^{*}\right)^{\theta_{i}-1} c_{i} a_{i i}\left(x_{i}-x_{i}^{*}\right)^{2} \\
& +\frac{1}{2} \sum_{i=1}^{n} \sum_{i \neq j}^{n} c_{i} a_{i j}\left(x_{i}^{*}-x_{i}\right)^{2} \\
& +\frac{1}{2} \sum_{i=1}^{n} \sum_{i \neq j}^{n} c_{i} a_{i j}\left(x_{j}^{*}-x_{j}\right)^{2}+\frac{1}{2} \sum_{i=1}^{n} c_{i} \sigma_{i}^{2} x_{i}^{*} \\
= & -\sum_{i=1}^{n}\left(x_{i}^{*}\right)^{\theta_{i}-1} c_{i} a_{i i}\left(x_{i}-x_{i}^{*}\right)^{2} \\
& +\frac{1}{2} \sum_{i=1}^{n} \sum_{i \neq j}^{n} c_{i} a_{i j}\left(x_{i}^{*}-x_{i}\right)^{2} \\
& +\frac{1}{2} \sum_{i=1}^{n} \sum_{i \neq j}^{n} c_{j} a_{j i}\left(x_{i}^{*}-x_{i}\right)^{2}+\frac{1}{2} \sum_{i=1}^{n} c_{i} \sigma_{i}^{2} x_{i}^{*} \\
= & -\sum_{i=1}^{n}\left[c_{i}\left(x^{*}\right)^{\theta_{i}-1} a_{i i}-\frac{1}{2} \sum_{i \neq j}^{n}\left(c_{i} a_{i j}+c_{j} a_{j i}\right)\right] \\
& \times\left(x_{i}^{*}-x_{i}\right)^{2}+\frac{1}{2} \sum_{i=1}^{n} c_{i} \sigma_{i}^{2} x_{i}^{*} . \\
& \\
&
\end{aligned}
$$

Note that $\left(c_{i}\left(x^{*}\right)^{\theta_{i}-1} a_{i i}-\sum_{i \neq j}\left(c_{i} a_{i j}+c_{j} a_{j i}\right)\right)>0, i=1, \ldots, n$, and

$$
\begin{aligned}
& \frac{1}{2} \sum_{k=1}^{n} c_{i} x_{i}^{*} \sigma_{i}^{2} \\
& \quad<\min _{1 \leq i \leq n}\left\{\left(x_{i}^{*}\right)^{2}\left(c_{i}\left(x^{*}\right)^{\theta_{i}-1} a_{i i}-\sum_{i \neq j}\left(c_{i} a_{i j}+c_{j} a_{j i}\right)\right)\right\} .
\end{aligned}
$$

Then the ellipsoid

$$
\begin{aligned}
& \sum_{i=1}^{n}\left[c_{i}\left(x^{*}\right)^{\theta_{i}-1} a_{i i}-\frac{1}{2} \sum_{i \neq j}^{n}\left(c_{i} a_{i j}+c_{j} a_{j i}\right)\right]\left(x_{i}^{*}-x_{i}\right)^{2} \\
& \quad=\frac{1}{2} \sum_{i=1}^{n} c_{i} \sigma_{i}^{2} x_{i}^{*},
\end{aligned}
$$

lies entirely in $R_{+}^{n}$. Let $U \subset \bar{U} \subset R_{+}^{n}$ be a neighborhood of the ellipsoid such that, for any $x \in R_{+}^{n^{+}} \backslash U, \mathscr{L} V<0$. We therefore have verified condition (ii) in Lemma 5. 
Now we begin to verify condition (i) in Lemma 5. It is easy to see that $\lambda_{\text {min }}\left(H^{T}(x) H(x)\right) \geq 0$. If $\lambda_{\text {min }}\left(H^{T}(x) H(x)\right)=$ 0 , then there exists $\xi \neq 0$ such that $\xi^{T} H^{T}(x) H(x) \xi=0$. This implies that $\left(\operatorname{diag}\left(\sigma_{1} x_{1}, \ldots, \sigma_{n} x_{n}\right) \xi=0\right.$. Then we have $\xi=0$, which contradicts the fact that $\xi \neq 0$. Noting that $\lambda_{\text {min }}\left(H^{T}(x) H(x)\right)$ is a continuous function of $x \in \bar{U}$, we therefore have

$$
\min _{x \in \bar{U}} \lambda_{\min }\left(H^{T}(x) H(x)\right)>0 .
$$

This immediately implies condition (i) in Lemma 5. The proof is completed.

Now we denote by $\mu(\cdot)$ the stationary distribution. The mean vector of $\mu(\cdot)$ is important and useful information on population systems, from which we can infer asymptotically the mean of $x_{i}(t)$ and the size of each species. If we can show that $\int_{R_{+}^{n}}|z| \mu(d z)<\infty$, then the mean vector $\bar{\mu}=$ $\left(\bar{\mu}_{1}, \ldots, \bar{\mu}_{n}\right)^{T}$ is well defined. In this case, the ergodic theory stated above implies that

$$
\bar{\mu}_{i}:=\lim _{t \rightarrow \infty} \frac{1}{t} \int_{0}^{t} x_{i}(s) d s, \quad i=1, \ldots, n, \text { a.s. }
$$

Theorem 8. Let assumptions in Theorems 4 and 7 hold. Then

$$
a_{i i}\left(\bar{\mu}_{i}\right)^{\theta_{i}}+\sum_{j \neq i} a_{i j} \bar{\mu}_{j} \leq b_{i}-\frac{\sigma_{i}^{2}}{2}, \quad i=1, \ldots, n .
$$

Proof. The proof is composed of two parts. The first part is to show the well-definition of $\mu$ by dominated control convergence theorem. The second part is to prove assertion (45). Let $x(t)=x\left(t, x_{0}\right)$ for simplicity.

By the ergodic property of stationary distribution, for $m>$ $0, p>0$, we have

$$
\begin{array}{r}
\lim _{t \rightarrow \infty} \frac{1}{t} \int_{0}^{t}\left(x_{i}^{p}(s) \wedge m\right) d s=\int_{0}^{\infty}\left(z_{i}^{p} \wedge m\right) \mu(d y), \\
i=1, \ldots, n, \text { a.s. }
\end{array}
$$

The dominated convergence theorem yields that

$$
\begin{array}{r}
E\left[\lim _{t \rightarrow \infty} \frac{1}{t} \int_{0}^{t}\left(x_{i}^{p}(s) \wedge m\right) d s\right]=\frac{1}{t} \int_{0}^{t} E\left[\left(x_{i}^{p} \wedge m\right)\right] d s \\
i=1, \ldots, n .
\end{array}
$$

It follows from Lemma 2 that

$$
\int_{0}^{\infty}\left(z_{i}^{p} \wedge m\right) \mu(d z) \leq K_{p}, \quad i=1, \ldots, n .
$$

Letting $m \rightarrow \infty$ yields

$$
\int_{0}^{\infty} z_{i}^{p} \mu(d y) \leq K_{p}, \quad i=1, \ldots, n .
$$

That is to say, for any $p>0$, the functions $y^{p}$ are integrable with respect to the measure $\mu(\cdot)$. The well-definition of $\mu$ follows by letting $p=1$ in (49) straightforward.
Now we process to show assertion (45). For $i=1, \ldots, n$, simple computation shows that

$$
\begin{aligned}
\frac{\log x_{i}(t)}{t}= & \frac{\log x_{i}(0)}{t} \\
& +\frac{1}{t} \int_{0}^{t}\left[b_{i}-\frac{\sigma_{i}^{2}}{2}-a_{i i} x_{i}^{\theta_{i}}-\sum_{j=1, j \neq i}^{n} a_{i j} x_{j}(s)\right] d s \\
& +\frac{1}{t} \int_{0}^{t} \sigma_{i} d B_{i}(s) .
\end{aligned}
$$

The well-known Hölder inequality yields

$$
\int_{0}^{t} x_{i}(s) d s \leq\left(\int_{0}^{t} x_{i}^{\theta_{i}}(s) d s\right)^{1 / \theta_{i}}\left(\int_{0}^{t} 1 d s\right)^{1 / \theta_{i}^{\prime}} \quad i=1, \ldots, n,
$$

where $1 / \theta_{i}^{\prime}=1-1 / \theta_{i}$. This implies

$$
\int_{0}^{t} x_{i}(s) d s \leq\left(\int_{0}^{t} x_{i}^{\theta_{i}}(s) d s\right)^{1 / \theta_{i}} t^{1-1 / \theta_{i}}, \quad i=1, \ldots, n .
$$

The well-known Hölder inequality yields

$$
\frac{1}{t} \int_{0}^{t} x_{i}(s) d s \leq\left(\frac{1}{t} \int_{0}^{t} x_{i}^{\theta_{i}}(s) d s\right)^{1 / \theta_{i}}, \quad i=1, \ldots, n .
$$

This implies

$$
\begin{array}{r}
\frac{\log x_{i}(t)}{t} \leq \frac{\log x(0)}{t}+b_{i}-\frac{\sigma_{i}^{2}}{2}-a_{i i}\left(\frac{1}{t} \int_{0}^{t} x_{i}(s) d s\right)^{\theta_{i}} \\
-\frac{1}{t} \int_{0}^{t} \sum_{j=1, j \neq i}^{n} a_{i j} x_{j}(s) d s+\frac{1}{t} \int_{0}^{t} \sigma_{i} d B_{i}(s), \\
i=1, \ldots, n .
\end{array}
$$

By the law of strong large numbers for martingales and Theorem 4 , letting $t \rightarrow \infty$ on both sides of (54) yields

$$
a_{i i}\left(\bar{\mu}_{i}\right)^{\theta_{i}}+\sum_{j \neq i} a_{i j} \bar{\mu}_{j} \leq b_{i}-\frac{\sigma_{i}^{2}}{2}, \quad i=1, \ldots, n \text {. }
$$

which is the required assertion (45).

\section{Extinction}

One of the most basic questions one can ask in population dynamics is extinction, which means a species will be doomed. The interesting question is can the exponential extinction rate be estimated precisely? In many cases, we need to know the extinction rate of the species in order to have a suitable policy in investment and to have timely measures to protect them from the extinct disaster. 
Theorem 9. Let condition (5) and $\sigma_{i}^{2}>2 b_{i}, i=1, \ldots, n$, hold and $x\left(t, x_{0}\right)$ be the global solution to system (4) with any positive initial value $x_{0}$. Then the solution $x_{i}\left(t, x_{0}\right)$ to system (4) has the property that

$$
\lim _{t \rightarrow \infty} \frac{\log x_{i}\left(t, x_{0}\right)}{t}=-\left(\frac{\sigma_{i}^{2}}{2}-b_{i}\right), \quad i=1, \ldots, n, \text { a.s. }
$$

That is, the population will become extinct exponentially with probability one and the exponential extinction rate of the ith species is $-\left(\sigma_{i}^{2} / 2-b_{i}\right)$.

Proof. Let $x(t)=x\left(t, x_{0}\right)$ for simplicity. It follows from Itô's formula that

$$
\begin{aligned}
\log x_{i}(t)= & \log x_{i}(0)+\int_{0}^{t}\left(b_{i}-\frac{\sigma_{i}^{2}}{2}\right) d s \\
& -\int_{0}^{t}\left(a_{i i} x^{\theta_{i}}(s)+\sum_{i \neq j} a_{i j} x_{j}(s)\right) d s \\
& +\int_{0}^{t} \sigma_{i} d B_{i}(s), \quad i=1, \ldots, n,
\end{aligned}
$$

where $M_{i}(t)=\int_{0}^{t} \sigma_{i} d B_{i}(s)$ is the real-valued continuous local martingale vanishing at $t=0$, with the quadratic variation $\left\langle M_{i}(t), M_{i}(t)\right\rangle=\sigma_{i}^{2} t$. Dividing both sides by $t$ yields

$$
\begin{aligned}
\frac{\log x_{i}(t)}{t}= & \frac{\log x_{i}(0)}{t}+\frac{1}{t} \int_{0}^{t}\left(r-\frac{\sigma_{i}^{2}}{2}\right) d s \\
& -\frac{1}{t} \int_{0}^{t}\left(a_{i i} x^{\theta_{i}}(s)+\sum_{i \neq j} a_{i j} x_{j}(s)\right) d s \\
& +\frac{1}{t} \int_{0}^{t} \sigma_{i}^{2} d B_{i}(s), \quad i=1, \ldots, n .
\end{aligned}
$$

Using the law of strong large numbers for martingales (see [17]), we can claim that

$$
\lim _{t \rightarrow \infty} \frac{1}{t} \int_{0}^{t} \sigma_{i} d B_{i}(s)=0, \quad i=1, \ldots, n, \text { a.s. }
$$

Letting $t \rightarrow \infty$ yields

$$
\lim _{t \rightarrow \infty} \sup \frac{\log x_{i}(t)}{t} \leq-\left(\frac{\sigma_{i}^{2}}{2}-b_{i}\right), \quad i=1, \ldots, n \text {, a.s. }
$$

This shows that, for any $1 \leq i \leq k$ and $\epsilon \in\left(0, \min _{1 \leq i \leq k}\left\{\sigma_{i} / 2-\right.\right.$ $\left.b_{i}\right\}$ ), there is a positive random variable $T(\epsilon)$ such that, with probability one,

$$
x_{i}(t) \leq e^{-\left(\sigma_{i}^{2} / 2-b_{i}\right) t+\epsilon t}, \quad \forall t>T(\epsilon), i=1, \ldots, n, \text { a.s. }
$$

It follows that

$$
x_{i}^{\theta_{i}}(t) \leq e^{-\alpha\left(\sigma_{i}^{2} / 2-b_{i}\right) t+\alpha \epsilon t}, \quad \forall t>T(\epsilon), i=1, \ldots, n, \text { a.s. },
$$
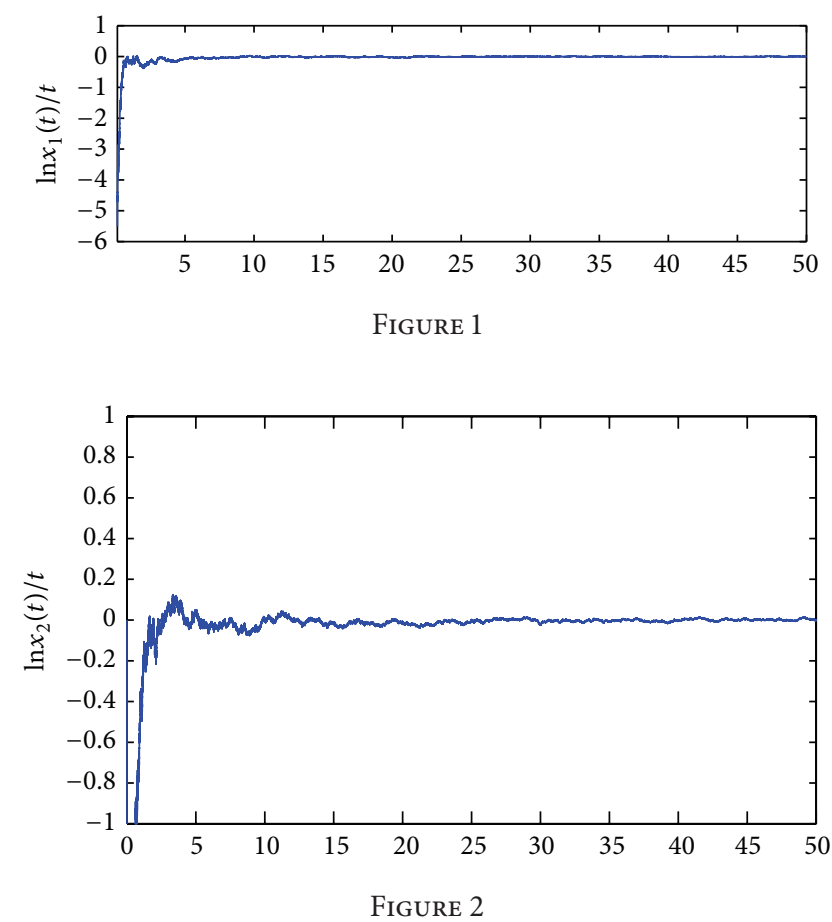

which means

$$
\begin{array}{r}
a_{i i} \int_{0}^{\infty} x_{i}^{\theta_{i}}(s) d s+\sum_{j \neq i} a_{i j} \int_{0}^{\infty} x_{j}^{\theta_{j}}(s) d s<\infty, \\
i=1, \ldots, n, \text { a.s. }
\end{array}
$$

The required assertion (58) follows by letting $t \rightarrow \infty$ on both sides of (54).

Remark 10. Theorem 9 showed that when the perturbation is large in the sense that $\sigma_{i}^{2}>2 b_{i}, i=1, \ldots, n$, the population will be forced to expire. And the exponential extinction rate is given precisely in terms of system's coefficients.

\section{Numerical Simulations}

In this section, to illustrate the usefulness and flexibility of the theorem developed in previous section, we present a numerical example.

Example 11. Consider a 2-dimensional stochastic GilpinAyala system as follows:

$$
\begin{aligned}
& d x_{1}=x_{1}\left(1-0.8 x_{1}^{1.5}-0.3 x_{2}\right) d t+\sigma x_{1} d B_{1}(t), \\
& d x_{2}=x_{2}\left(1-x_{2}^{1.2}-0.2 x_{1}\right) d t+\sigma x_{2} d B_{2}(t) .
\end{aligned}
$$

System (64) is exactly system (4) with $a_{11}=0.8>0, a_{12}=$ $0.3>0, a_{21}=0.2>0, a_{22}=1>0, b_{1}=1>0, b_{2}=1.2>0$, and $\theta_{1}=1.2, \theta_{2}=1.5$. We compute that $x_{1}^{*}=0.9109$ and $x_{2}^{*}=1.0148$. The existence and uniqueness of the solution follows from Lemma 1 . We consider the solution $x\left(t, x_{0}\right)$ with initial data $x_{1}(0)=0.5$ and $x_{2}(0)=0.5$. Let $x(t)=x\left(t, x_{0}\right)$ for simplicity. 


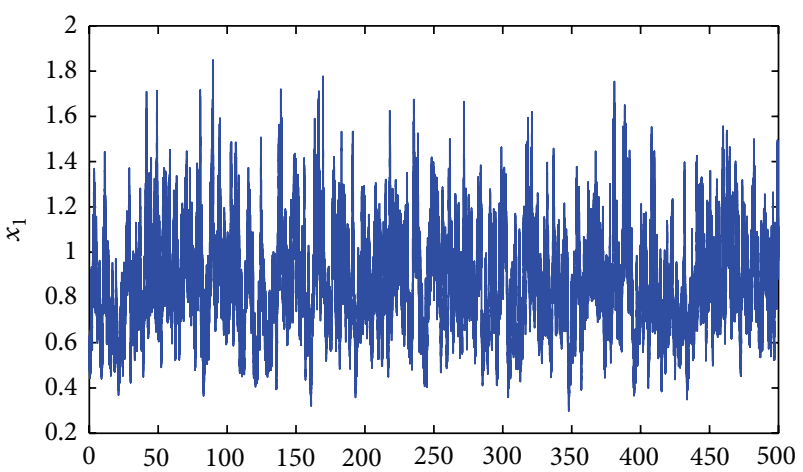

FIGURE 3

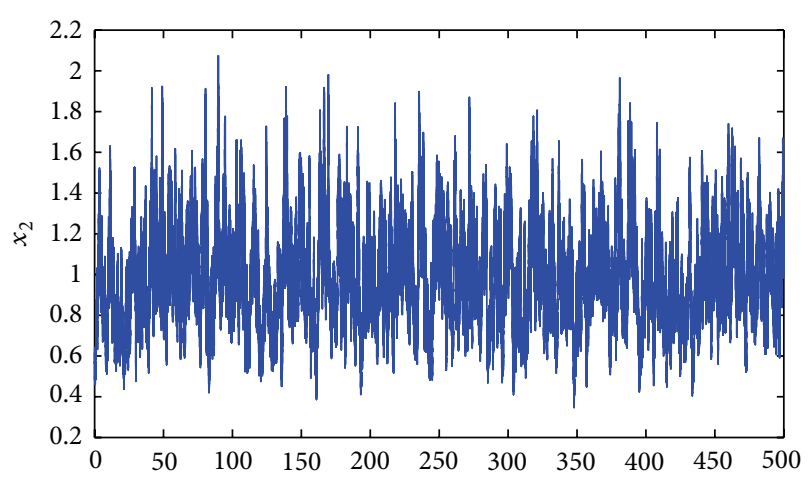

FigURE 4

(i) $\sigma=0.4$ : simple computation shows that

$$
\begin{gathered}
b_{1}-\frac{\sigma^{2}}{2}=0.92>0, \quad b_{2}-\frac{\sigma^{2}}{2}=1.12>0, \\
b_{1}-\frac{\sigma^{2}}{2}-a_{12}\left(b_{2}-\frac{\sigma^{2}}{2}\right)^{1 / \theta_{2}}=0.5903>0, \\
b_{2}-\frac{\sigma^{2}}{2}-a_{21}\left(b_{1}-\frac{\sigma^{2}}{2}\right)^{1 / \theta_{1}}=0.9308>0 .
\end{gathered}
$$

By Theorem 4, the solution to system (64) has the following property

$$
\lim _{t \rightarrow \infty} \frac{\log x_{1}(t)}{t}=0 ; \quad \lim _{t \rightarrow \infty} \frac{\log x_{2}(t)}{t}=0, \quad \text { a.s. }
$$

Figures 1 and 2 show the stochastic trajectories of $\log x_{1}(t) / t$ and $\log x_{2}(t) / t$ generated by the Heun scheme for time step $\Delta=10^{-3}$ for system $(64)$ on $[0,50]$, respectively.

Choosing $c_{1}=1$ and $c_{2}=0.5$, we further compute that

$$
\begin{gathered}
c_{1} a_{11}\left(x_{1}^{*}\right)^{0.5}-\frac{1}{2}\left(c_{1} a_{12}+c_{2} a_{21}\right)=0.5635>0, \\
c_{2} a_{22}\left(x_{2}^{*}\right)^{0.2}-\frac{1}{2}\left(c_{1} a_{12}+c_{2} a_{21}\right)=0.3015>0, \\
c_{1} \sigma\left(x_{1}^{*}\right)^{2}+c_{2} \sigma\left(x_{2}^{*}\right)^{2}=0.2837<\min \{0.5635,0.3015\} .
\end{gathered}
$$
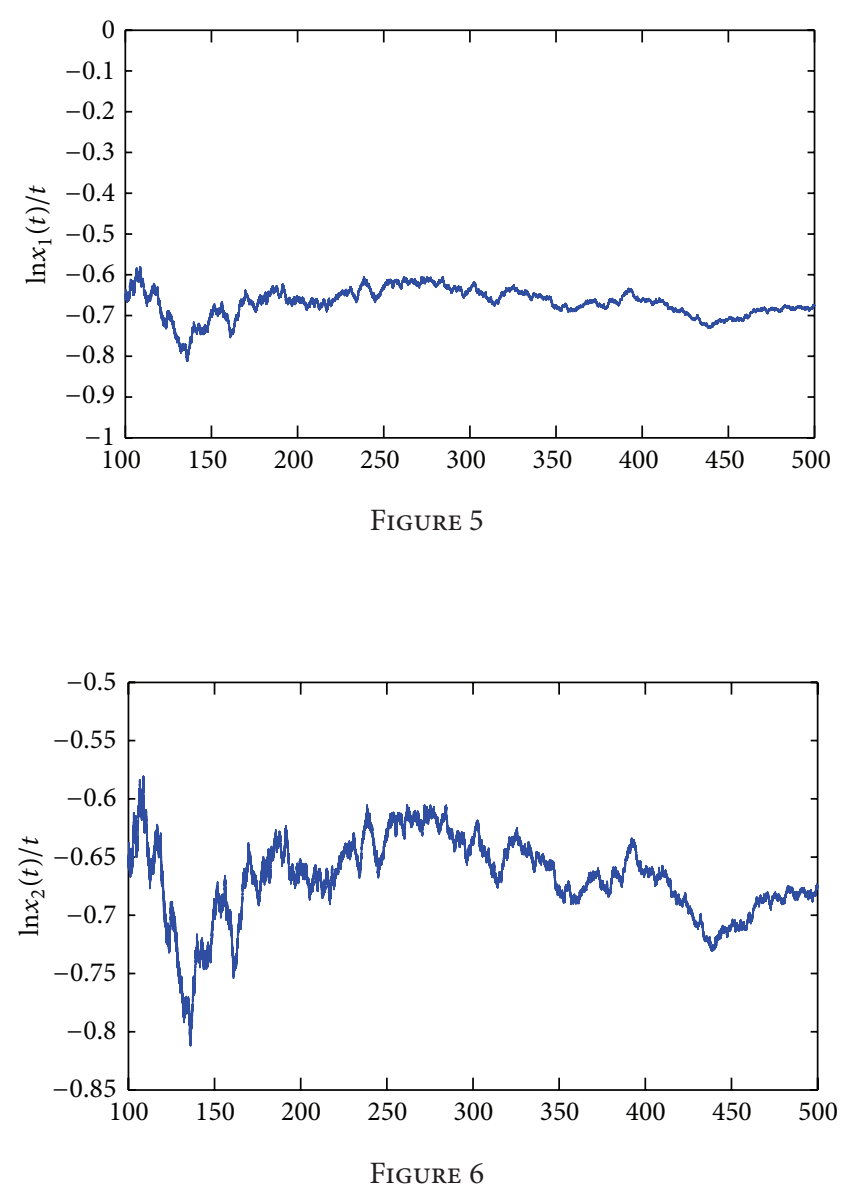

By virtue of Theorem 7 , system (64) has a unique stationary distribution. Figures 3 and 4 show the stochastic trajectories of $x_{1}(t)$ and $x_{2}(t)$ generated by the Heun scheme for time step $\Delta=10^{-3}$ for system (64) on $[0,500]$, respectively.

(ii) Consider $\sigma=2$.

Note that $1<2^{2} / 2,1.2<2^{2} / 2$, by virtue of Theorem 9 , system (64) is exponentially extinctive. Figures 5 and 6 show the stochastic trajectories of $\log x_{1}(t) / t$ and $\log x_{2}(t) / t$ generated by the Heun scheme for time step $\Delta=10^{-3}$ for system $(64)$ on $[100,500]$, respectively.

\section{Conclusion}

In this paper, we have investigated the asymptotic behavior for the stochastic Gilpin-Ayala competition system. Firstly, by utilizing stochastic analysis techniques and the stochastic comparison principle, the larger time behavior $\log x_{i}(t) / t, i=$ $1, \ldots, n$. has been researched. Secondly, by applying some techniques to deal with the nonquadratic item, sufficient conditions are obtained under which there is a stationary distribution to the system. Based on the condition, the estimation on the mean of the stationary distribution is presented. Finally, the sufficient criteria for extinction are established. 


\section{Conflict of Interests}

The authors declare that there is no conflict of interests regarding the publication of this paper.

\section{Acknowledgment}

This work was jointly supported by the National Natural Science Foundation of China (Grant nos. 61304070, 11271146, and 61374080), the National Key Basic Research Program of China (973 Program) (2013CB228204), the Natural Science Foundation of Zhejiang Province (LY12F03010), the Fundamental Research Funds for the Central Universities of China (Grant no. 2013B00614), and a project funded by the Priority Academic Program Development of Jiangsu Higher Education Institutions.

\section{References}

[1] M. E. Gilpin and F. G. Ayala, "Global models of growth and competition," Proceedings of the National Academy of Sciences of the United States of America, vol. 70, pp. 3590-3593, 1973.

[2] D. Jiang and N. Shi, "A note on nonautonomous logistic equation with random perturbation," Journal of Mathematical Analysis and Applications, vol. 303, no. 1, pp. 164-172, 2005.

[3] D. Jiang, N. Shi, and X. Li, "Global stability and stochastic permanence of a non-autonomous logistic equation with random perturbation," Journal of Mathematical Analysis and Applications, vol. 340, no. 1, pp. 588-597, 2008.

[4] C. Y. Ji, D. Q. Jiang, and N. Z. Shi, "Analysis of a predator-prey model with modified Leslie-Gower and Holling-type II schemes with stochastic perturbation," Journal of Mathematical Analysis and Applications, vol. 359, no. 2, pp. 482-498, 2009.

[5] X. Li, A. Gray, D. Jiang, and X. Mao, "Sufficient and necessary conditions of stochastic permanence and extinction for stochastic logistic populations under regime switching," Journal of Mathematical Analysis and Applications, vol. 376, no. 1, pp. 1128, 2011.

[6] M. Liu and K. Wang, "Persistence and extinction in stochastic non-autonomous logistic systems," Journal of Mathematical Analysis and Applications, vol. 375, no. 2, pp. 443-457, 2011.

[7] X. Mao, C. Yuan, and J. Zou, "Stochastic differential delay equations of population dynamics," Journal of Mathematical Analysis and Applications, vol. 304, no. 1, pp. 296-320, 2005.

[8] X. Mao, S. Sabanis, and E. Renshaw, "Asymptotic behaviour of the stochastic Lotka-Volterra model," Journal of Mathematical Analysis and Applications, vol. 287, no. 1, pp. 141-156, 2003.

[9] C. Zhu and G. Yin, "On hybrid competitive Lotka-Volterra ecosystems," Nonlinear Analysis: Theory, Methods \& Applications, vol. 71, no. 12, pp. e1370-e1379, 2009.

[10] C. Zhu and G. Yin, "On competitive Lotka-Volterra model in random environments," Journal of Mathematical Analysis and Applications, vol. 357, no. 1, pp. 154-170, 2009.

[11] X. Mao, "Stationary distribution of stochastic population systems," Systems \& Control Letters, vol. 60, no. 6, pp. 398-405, 2011.

[12] D. Jiang, C. Ji, X. Li, and D. O'Regan, "Analysis of autonomous Lotka-Volterra competition systems with random perturbation," Journal of Mathematical Analysis and Applications, vol. 390, no. 2, pp. 582-595, 2012.
[13] M. Liu and K. Wang, "Stationary distribution, ergodicity and extinction of a stochastic generalized logistic system," Applied Mathematics Letters, vol. 25, no. 11, pp. 1980-1985, 2012.

[14] H. Liu, X. Li, and Q. Yang, "The ergodic property and positive recurrence of a multi-group Lotka-Volterra mutualistic system with regime switching," Systems \& Control Letters, vol. 62, no. 10, pp. 805-810, 2013.

[15] N. Ikeda Wantanabe, Stochastic Differential Equations and Diffusion Processes, North-Holland, Amsterdam, The Netherlands, 1981.

[16] R. Z. Hasminskii, Stochastic Stability of Differential Equations, Springer, Berlin, Germany, 2011.

[17] X. Mao and C. Yuan, Stochastic Differential Equations with Markovian Switching, Imperial College Press, London, UK, 2006. 


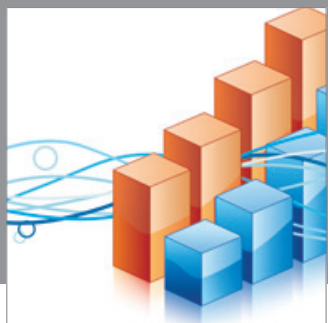

Advances in

Operations Research

mansans

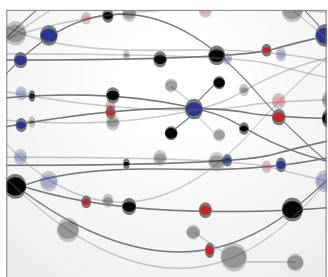

The Scientific World Journal
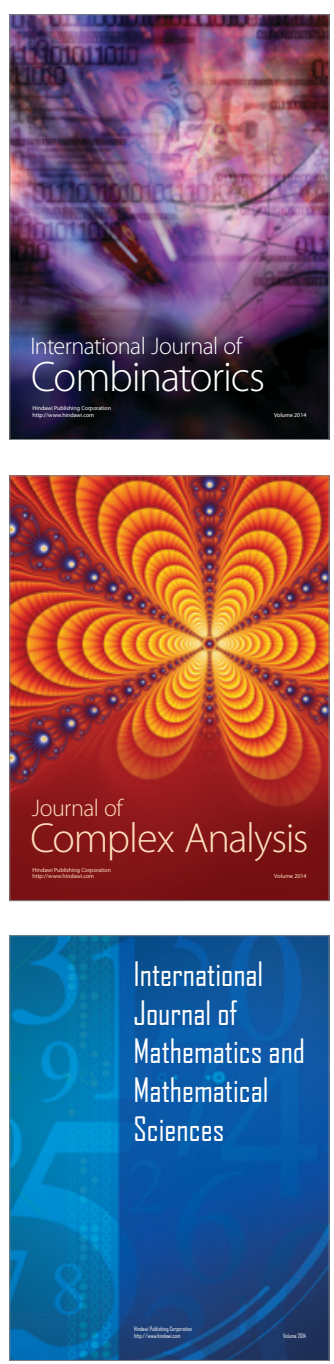
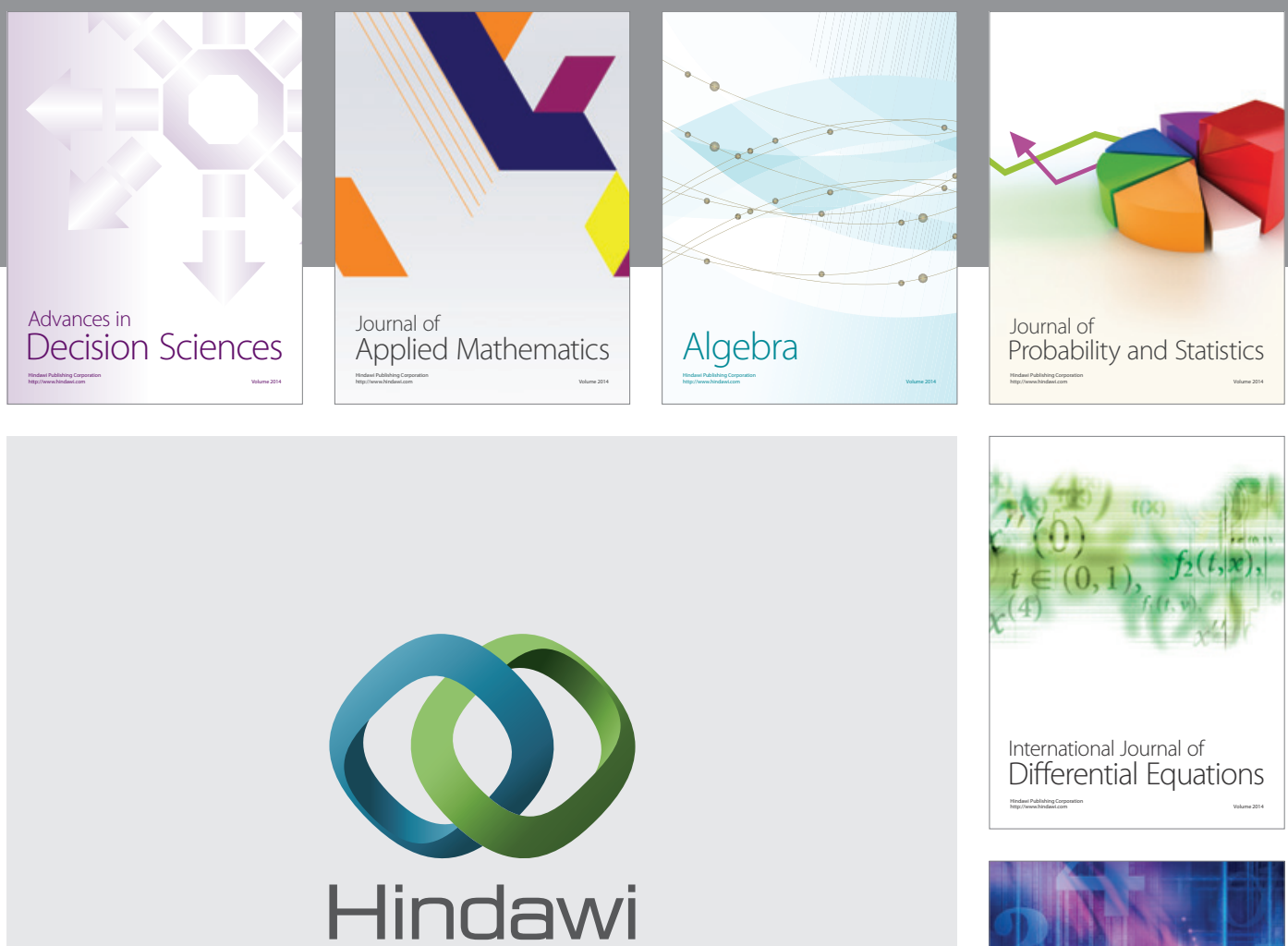

Submit your manuscripts at http://www.hindawi.com
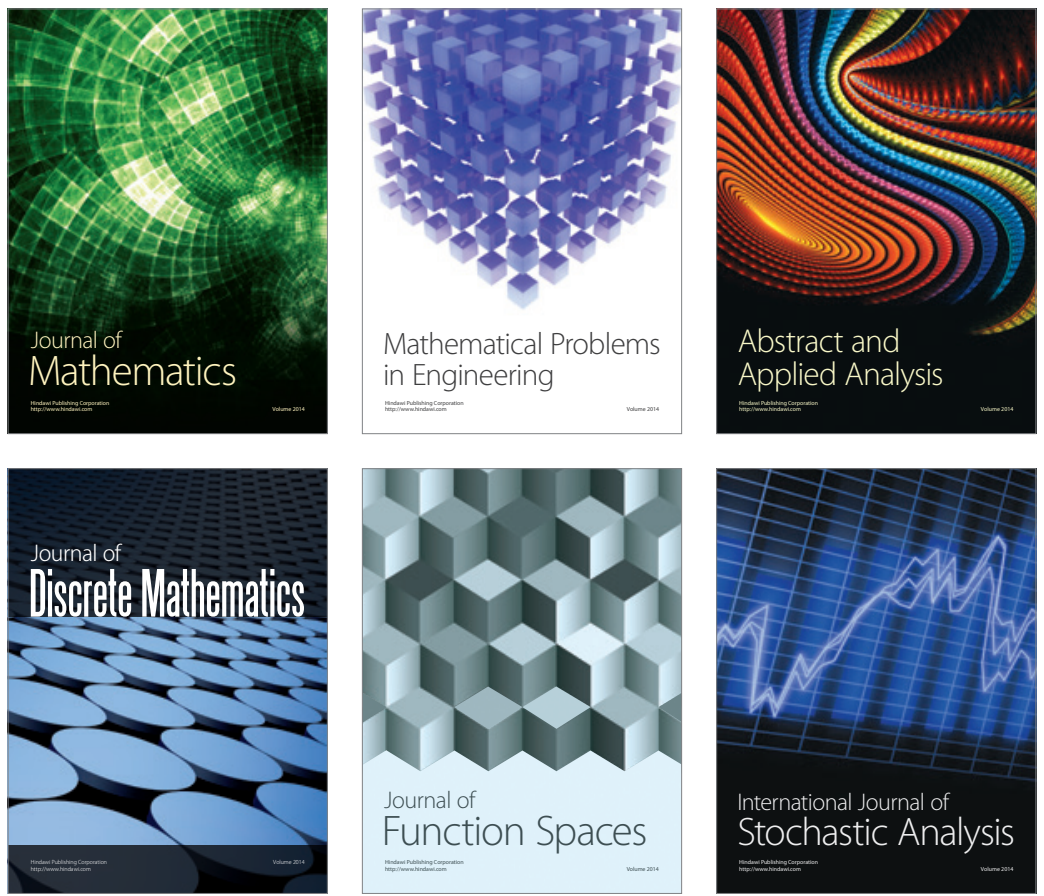

Journal of

Function Spaces

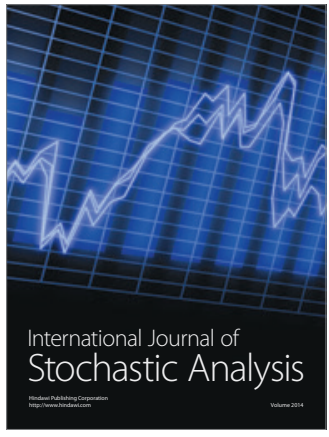

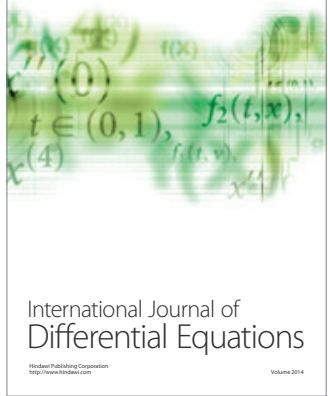
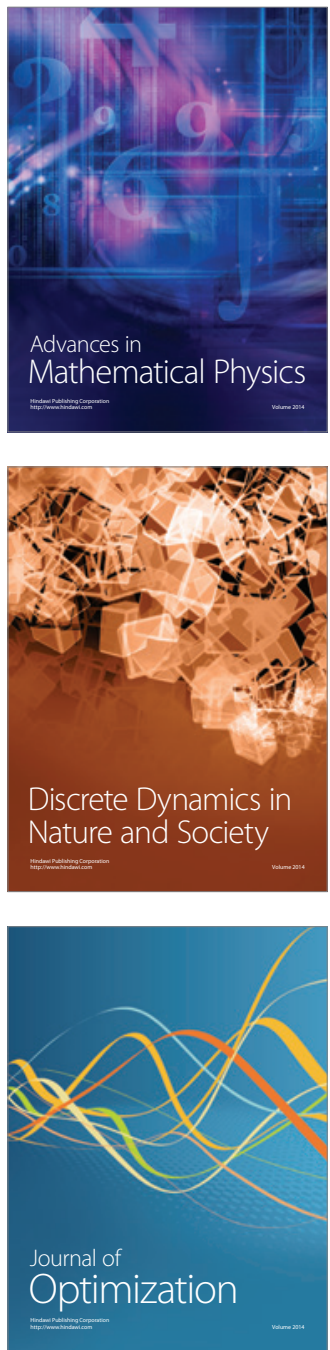